\title{
NUEVOS DATOS SOBRE ALGUNAS ESPECIES DE HEMERÓBIDOS DE LA PENÍNSULA IBÉRICA E ISLAS CANARIAS, INCLUYENDO UNA NUEVA ESPECIE INVASORA DE ORIGEN NEOTROPICAL EN PORTUGAL (INSECTA, NEUROPTERA, HEMEROBIIDAE)
}

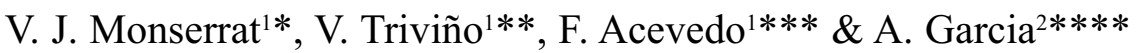

\section{RESUMEN}

V. J. Monserrat, V. Triviño, F. Acevedo \& A. Garcia. 2013. Nuevos datos sobre algunas especies de Hemeróbidos de la península Ibérica e islas Canarias, incluyendo una nueva especie invasora de origen neotropical en Portugal (Insecta, Neuroptera, Hemerobiidae). Grael/sia, 69(2): 157-168.

Se anotan nuevos datos sobre la distribución, biología y fenología de 25 especies de hemeróbidos (Insecta, Neuroptera: Hemerobiidae) de las Faunas Ibérica y Canaria.

La especie Hemerobius bolivari Banks, 1910, ampliamente distribuida por la Región Neotropical, se cita por primera vez de Europa como una nueva especie invasora, a partir de ejemplares recolectados en colonias de Thaumastocoris peregrinus Carpintero y Dellapé, 2006 (Hemiptera: Thaumastocoridae:) sobre Eucalyptus en Lisboa (Portugal). Esta especie, junto a Sympherobius gayi Navás, 1910, también originaria de Sudamérica, es la segunda especie invasora de origen neotropical que de esta familia se cita en la Península lbérica, en este caso también de Portugal. Se mencionan varias especies que han sido muy escasamente citadas en la Península lbérica, y entre ellas destaca Wesmaelius ravus (Withycombe, 1923), que se cita por segunda vez en la Península Ibérica, en este caso de Sierra Nevada (Granada).

A partir de muestreos realizados en el sur de España (Málaga, Granada), se desprende actividad en los imagos de Micromus angulatus (Stephens, 1836) en los meses de invierno (a lo largo de todo el año en la Península lbérica, incluyendo esta zona ibérica), y estos datos también parecen corroborar su vinculación con la vegetación arvense.

Palabras clave: Insecta; Neuroptera; Hemerobiidae; faunística; biología; fenología; Península Ibérica; España; Portugal; Islas Canarias; Paleártico; Neotropical.

\section{ABSTRACT}

V. J. Monserrat, V. Triviño, F. Acevedo \& A. Garcia. 2013. New data on some brown lacewings species from the lberian Peninsula and the Canary Islands, including a new Neotropical invasive species in Portugal. Grael/sia, 69(2): 157-168 (in Spanish).

New data on the distribution, biology and phenology of 25 brown lacewings species (Insecta, Neuroptera: Hemerobiidae) of Iberian and Canaries fauna are given.

The species Hemerobius bolivari Banks, 1910, widely distributed in the Neotropical Region, is recorded for the first time in Europe as a new invasive species, from specimens

\footnotetext{
Departamento de Zoología y Antropología Física, Facultad de Biología, Universidad Complutense, 28040 Madrid (España).

2 Centro de Estudos Florestais, Instituto Superior de Agronomia, Universidade de Lisboa, Tapada da Ajuda, 1349-017 Lisboa (Portugal)
}

* artmad@bio.ucm.es_** victor.trivi@gmail.com ***facevedoramos.f@hotmail.es_****andregarcia@isa.ulisboa.pt 
collected on Thaumastocoris peregrinus Carpintero \& Dellapé, 2006 (Hemiptera: Thaumastocoridae) colonies on Eucalyptus in Lisbon (Portugal). This brown lacewing species, along with Sympherobius gayi Navás, 1910, also known from South America, is the second invasive species of neotropical origin, belonging to this family, that is recorded in the Iberian Peninsula, both from Portugal. We also mention several species that have been seldom recorded in the Iberian Peninsula, and among them Wesmaelius ravus (Withycombe, 1923 ) is recorded for the second time in the Iberian Peninsula, now from Sierra Nevada (Granada).

From samplings made in southern Spain (Malaga, Granada) Micromus angulatus (Stephens, 1836) shows activity as imagoes during winter time (the entire year in the lberian Peninsula, including this area), and these data seem also to confirm its link to grass vegetation.

Key words: Insecta; Neuroptera; Hemerobiidae; Faunistics; Biology; Phenology; Iberian Peninsula; Spain; Portugal; Canary Islands; Palaearctic; Neotropical.

\section{Introducción}

Los hemeróbidos constituyen una interesante familia de neurópteros debido a su amplia distribución geográfica, al elevado número de individuos que frecuentemente constituyen sus poblaciones, $y$ especialmente por su utilización como agentes de control de pequeños artrópodos fitófagos (McEwen et al., 2001). No obstante, es proporcionalmente escasa la atención que vienen recibiendo en comparación con otras familias de neurópteros de interés en el control biológico, sea Chrysopidae o Coniopterygidae, y son proporcionalmente escasos los datos existentes sobre la distribución, la biología, fenología, morfología, estadios juveniles y variabilidad de la mayoría de las 600 especies que se consideran válidas en esta familia (Oswald, 1988, 1990, 1993a, b, 1994, 1996; Makarkin, 1990; Monserrat, 1990a, b, c, d, 1991, 1993, 1994, 1996, 1998, 2000, 2001, 2003, 2004, 2008a; Monserrat \& Deretsky, 1999; Hölzel, 2007, etc., por citar los más recientes).

A nivel genérico, su taxonomía y sistemática ha sido recientemente tratada (Oswald, 1993a). Sin embargo, su estado general de conocimiento es aún muy incompleto, siendo francamente escasos los trabajos de revisión global en la mayoría de sus géneros, son muchas las especies inadecuadamente descritas y tipificadas, otras no han sido citadas posteriormente desde su descripción original, y otras son muy parcialmente conocidas, siendo muy parco el conocimiento sobre su morfología, venación, genitalia y variabilidad en muchas especies, hecho que conlleva a errores de identificación y que provoca confusiones en su distribución geográfica real. Por ello, de forma global, puede decirse que la situación taxonómica y sistemática de las especies de esta familia es aún bastante incompleta y fragmentaria.
Sin duda, las faunas Paleártica, Neártica y Australiana son las mejor conocidas sobre este particular, si bien el límite real de distribución de muchas especies es, con frecuencia, impreciso o incompleto $\mathrm{y}$, en ocasiones, los datos sobre su biología son escasos y el nivel de conocimiento de las faunas de muchos países, incluso en zonas relativamente bien estudiadas como Europa y Estados Unidos es aún poco satisfactorio. Este hecho es aún más evidente en faunas de otros continentes, y nuevos trabajos faunísticos van aportando nuevos datos que van completando las lagunas existentes sobre su taxonomía, distribución, biología o estadios juveniles (Aspöck et al., 1980, 2001; Monserrat, 1980, 1984b, 1985, 1986, 1990a, b, c, d, 1991, 1992a, b, 1993, 1994, 1996, 1998, 2000, 2001, 2002, 2003, 2004; Klimaszewski \& Kevan, 1985, 1987a, b, 1988a, b, 1990a, b, c, 1992; Kevan \& Klimaszewski, 1986, 1987; New, 1988a, b; Oswald, 1988, 1990, 1993a, b, 1994, 1996; Makarkin, 1990, 1993; Wise, 1993, 2000; Monserrat, 1991; Marín \& Monserrat, 1995; Monserrat \& Marín, 1996; Monserrat \& Deretsky, 1999; Hölzel, 2007; Makarkin \& Monserrat, 2007, etc., por citar los más recientes).

En lo que respecta a la Fauna Ibero-Balear y Canaria, se ha venido aportando recientemente abundante información, aunque existen aún multitud de lagunas y de áreas completamente inexploradas sobre este grupo (Monserrat \& Triviño, 2013). A las especies de esta familia más comunes y habituales que históricamente fueron descritas o iban siendo conocidas en la Península Ibérica, se han venido añadiendo más recientemente nuevas opiniones y nuevos elementos (Wesmaelius reisseri, W. helveticus, $W$. ravus, $W$. fassnidgei, Sympherobius klapaleki, S. maculipennis, $S$. gayi, Psectra diptera, Drepanepteryx phalaenoides, Hemerobius perele- 


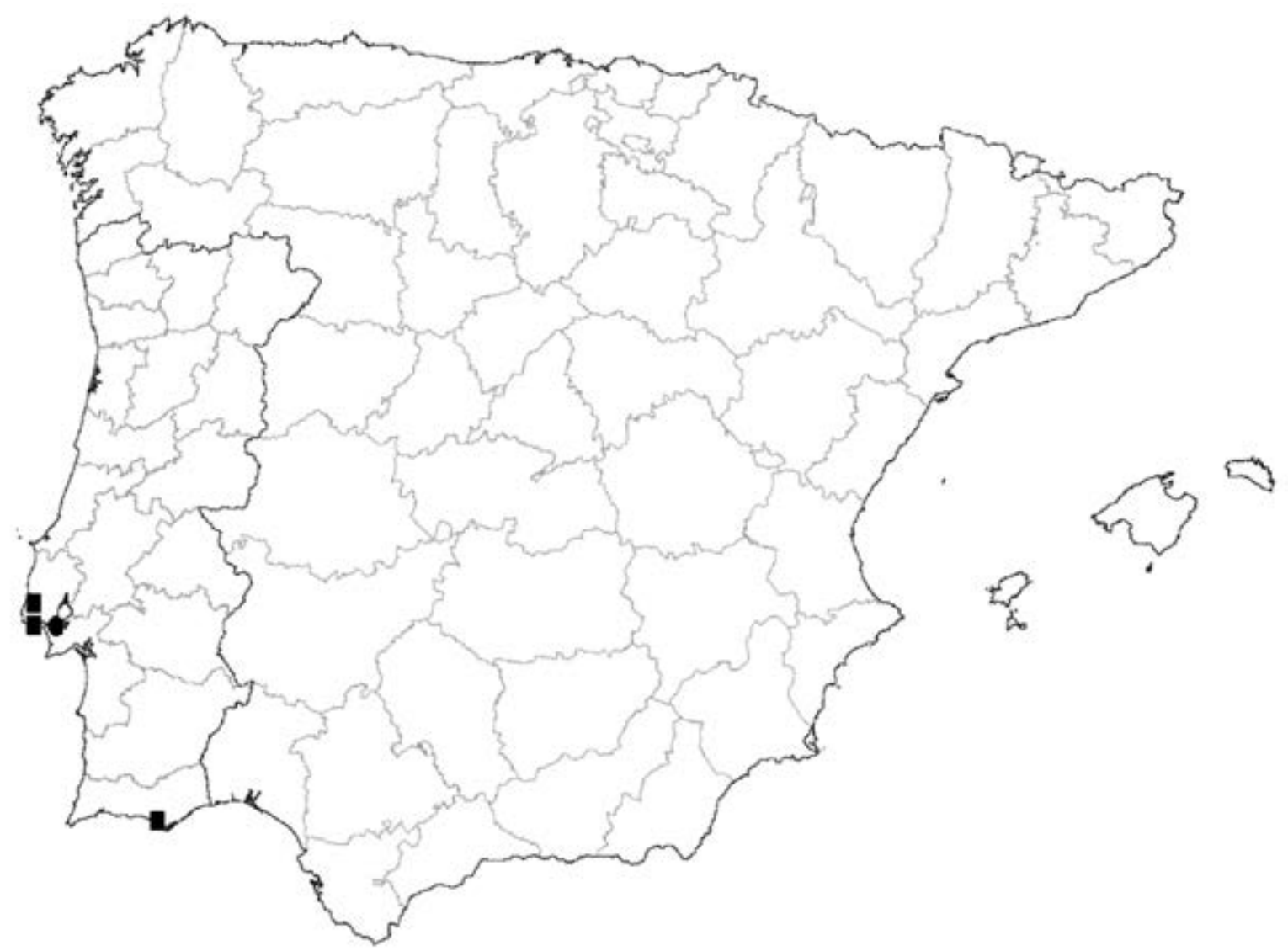

Fig. 1.- Distribución en la Península lbérica de las especies invasoras Hemerobius bolivari Banks, 1910 (•) y Sympherobius gayi Navás, $1910(\mathbf{\square})$.

Fig. 1.- Distribution in the Iberian Peninsula of the invasive species Hemerobius bolivari Banks, 1910 ( $\bullet$ ) and Sympherobius gayi Navás, 1910 (ם).

gans, etc.), con incluso alguna especie neotropical considerada/ble como especie invasora (Fig. 1), aparentemente asentada en Portugal (Monserrat, 1976a, b, 1978a, b, 1984a, 1985, 1986, 1990b, c, d, 1991, 1993, 1994, 2004, 2008a, 2010; Aspöck et al.,1980; Aspöck \& Aspöck, 1982; Monserrat \& Deretsky, 1999), citas que han acabado por conformar una de las familias más rica en especies (39) dentro de la Fauna Europea (52) (Aspöck et al., 1980; Monserrat $\&$ Triviño, 2013). Con respecto a la fauna de las Islas Baleares, Monserrat (2005) anota 15 especies, y pone en duda algunas citas antiguas, y con respecto a las Islas Canarias, son conocidas 9 especies $(\mathrm{Ohm}$ \& Báez, 2004; Báez \& Oromí, 2010).

Recientemente Monserrat (2011) ha resuelto la posición taxonómica de las especies ibéricas perte- necientes a esta familia que hasta entonces poseían una situación taxonómica dudosa o sin resolver, y la lista de las especies ibero-baleares de esta familia con sus sinonimias y combinaciones nomenclatoriales bajo las cuales han sido citadas, así como el cartografiado de su distribución hasta ahora conocida ha sido recientemente aportado por Monserrat \& Triviño (2013).

En el presente artículo anotamos nuevos datos sobre la distribución, biología y fenología de 25 especies que citamos de la Península Ibérica (España, Portugal) e Islas Canarias, (Tenerife), alguna de ellas muy poco citadas con anterioridad en estas zonas, y de las que una de ellas se cita por primera vez en la Fauna Europea de Portugal. 


\section{Material y método}

Salvo algunos ejemplares pertenecientes al Instituto Andaluz de Investigación y Formación Agraria, Laboratorio de Entomología Agraria, Málaga, España (IFAPA), el resto del material, permanece en la colección de V. J. Monserrat (Madrid, España), y salvo los ejemplares en los que se anota su recolector, el resto del material ha sido recolectado por los autores.

El material estudiado se cita ordenado alfabética y cronológicamente siguiendo la siguiente secuencia: país, provincia / isla, localidad de captura, coordenadas, altitud, fecha de captura, número de $\sigma^{\pi} \sigma^{\pi}$, de 우 ㅇ y/o de larvas estudiados, datos de biología, recolector (si no es alguno de los autores) y, en su caso, institución a la que dicho material pertenece, según las siglas anotadas. Los géneros de las plantas sobre las que se han hallado los ejemplares se citan apocopados, una vez han sido citados dentro de cada una de las especies mencionadas.

Para acceder a la genitalia de los ejemplares conservados en seco se reblandecía el extremo del abdomen con agua durante unas horas, y todos (conservados secos o en alcohol) se aclaraban con ácido láctico caliente $\mathrm{y}$, ocasionalmente, se ha empleado negro de clorazenol para acceder a las estructuras internas menos visibles.

Para la ordenación sistemática se sigue a Oswald (1993a). Para la distribución general de las especies se ha seguido a Aspöck et al. (1980, 2001), y sobre la preferencia de sustrato de las especies citadas puede consultarse Monserrat \& Marín (1996).

\section{Material estudiado}

Hemerobiidae Latreille, 1802

Hemerobiinae Latreille

Hemerobius humulinus Linnaeus, 1758

Elemento holártico extendido en América desde Alaska a Guatemala. Frecuente en zonas boscosas y húmedas, y citado sobre gran variedad de plantas, generalmente planifolios.

Material estudiado: ESPAÑA: Huesca, Bielsa, Circo de Pineta, 31TBH62 (1300 m), 18.VII.2009, 1 ㅇ sobre Corylus avellana, Villanova, Coll de Sahún, 31TBH91 (1300 m), 17.VII.2009, $20^{7} \sigma^{7}, 4$ 우 우 sobre $C$. avellana. Lérida, Es Bòrdes, 31TCH13 (830 m), 16.VII.2009, 1 우 en vegetación de rivera, La Bonaigua, Bosque del Gerdar, 31TCH32 (1472 m),
16.VII.2009, 1 ㅇ sobre C. avellana. Madrid, Chinchón, El Salitral, 30TVK64 (560 m), 20.VI.2009, 1 우 a la luz.

Hemerobius nitidulus Fabricius, 1777

Especie paleártica occidental. Asociada a coníferas.

Material estudiado: ESPAÑA: Madrid, El Escorial, Abantos, Fuente del Cervunal, 30TVK09 (1684 m), 7.VI.2008, $40^{\top} \sigma^{\top}, 6$ 우 우 sobre Pinus sylvestris, 21.VI.2008, 5 우 우 sobre P. sylvestris, 17.VII.2008, $10^{\top}$ sobre P. sylvestris, 31.VII.2008, $10^{\circ}, 1$ ㅇ sobre P. sylvestris, Arboreto Luis Ceballos, 30TVK09 (1317 m), 12.IX.2008, 1 ㅇ sobre P. sylvestris.

\section{Hemerobius micans Olivier, 1792}

Elemento paleártico occidental. Generalmente asociado a planifolios en medios húmedos, especialmente a Fagus.

Material estudiado: ESPAÑA: Huesca, Bielsa, Circo de Pineta, 31TBH62 (1300 m), 18.VII.2009, 1 ㅇ sobre Corylus avellana.

Hemerobius lutescens Fabricius, 1793

Especie paleártica occidental y central. Asociada a planifolios en medios húmedos.

Material estudiado: ESPAÑA: Asturias, Luarca, 29TPJ92 (10 m), 2.VII.2011, 1 우 . Pontevedra, Fontán, 29TNH30 (194 m), 21.IX.2009, $20^{\pi} \sigma^{\pi}$ sobre Quercus robur.

Hemerobius stigma Stephens, 1836

Especie holártica, introducida en Hawaii. Mayoritariamente asociada a coníferas.

Material estudiado: ESPAÑA: Álava, Zuia, P.N. Gorbeia, 30TWN16 $(800 \mathrm{~m}), 23 . V I .2007,20^{\top} \sigma^{\pi}, 1$ 우 sobre Pinus nigra, S.Pagola. Ciudad Real, Navas de Estena, P.N. de Cabañeros, 30SUJ67 $(660 \mathrm{~m}), 16 . \mathrm{V} .2012,1$ 우 a la luz. Granada, Collado de la Sabina, 30SVG60 (2050 m), 30.VI.2011, 1 ㅇ sobre P. sylvestris, 25.VIII.2011, $20^{\top} \sigma^{\top}$ sobre P. sylvestris, 10.VII.2012, $30^{7} \sigma^{7}, 6$ 우 우 sobre P. sylvestris, Hoya de la Mora, 30SVG60 (2200 m), 29.VI.2011, $10^{7}$ sobre P. sylvestris. Madrid, El Escorial, Abantos, 30TVK09 (1600 $\mathrm{m}), 4$.VII.2012, 2 우 우 sobre P. sylvestris.

Hemerobius gilvus Stein, 1863

Elemento mediterráneo septentrional, probablemente holomediterráneo. Generalmente asociado a planifolios del género Quercus en medios relativamente húmedos. 
Material estudiado: ESPAÑA: Ciudad Real, Navas de Estena, P.N. de Cabañeros, 30SUJ67 (660 m), 16.V.2012, $10^{7}$ sobre Quercus ilex, 17.V.2012, $10^{7}$ sobre Q. pyrenaica.

\section{Hemerobius eatoni Morton, 1906}

Especie endémica de Canarias (Tenerife, La Palma, Gran Canaria, El Hierro, La Gomera). Generalmente asociada a coníferas (Pinus canariensis).

Material estudiado: ESPAÑA: Canarias, Tenerife, Barranco del Agua, 28RCS31 (900 m), 13.VII.2008, $20^{7} \sigma^{7}$, 3 우 우 sobre Viburnum rigidum, E. Fernández, Mirador de Chipeque, P. Natural Corona Forestal, 28RCS53 (1899 m), 4.VIII.2008, $10^{\pi}, 1$ ㅇ sobre Pinus canariensis, P.N. del Teide, Mirador La Crucita, 28RCS53 (2070 m), 4.VIII.2008, 3 우 우 sobre $P$. canariensis.

\section{Hemerobius bolivari Banks, 1910}

Especie descrita de Colombia por Banks (1910), siendo un elemento frecuente, abundante, y en origen ampliamente distribuido por la Región Neotropical, desde México, Costa Rica, Guatemala y Panamá a Tierra del Fuego, Islas Malvinas y Archipiélago de Juan Fernández (Monserrat, 1996).

Se trata de una especie de morfología, tamaño y pigmentación relativamente variable, muy eurioica en la selección de hábitat y sustrato vegetal, y de amplio rango altitudinal (10-3100 m) (Monserrat, 1996, 2002, 2003, 2008a; Lara \& Freitas, 2003). Su morfología, variabilidad y biología fue revisada por Monserrat $(1996,1998)$, y sobre sus estadios preimaginales, Monserrat (2008b) describió la ultraestructura de su huevo, y sus fases juveniles fueron descritas por Reguilón (2002) y Monserrat (2003).

Los ejemplares ahora citados fueron recolectados en fase de larva sobre hojas de Eucalyptus situados a la intemperie en el Arboreto del Instituto Superior de Agronomía de Lisboa (Garcia et al., 2013), y estas larvas fueron alimentadas en el laboratorio hasta obtener los correspondientes imagos que ahora se citan. Se da el caso de que estos árboles estaban infestados por una plaga de hemípteros (Hemiptera: Thaumastocoridae: Thaumastocoris peregrinus Carpintero y Dellapé 2006), insecto conocido con el nombre común de chinche del eucalipto. Este gregario, nocivo y pequeño insecto (2-4 $\mathrm{mm}$ de longitud) se alimenta de savia (Cassis, Schuh \& Brailovsky, 1999; Noack \& Rose, 2007; Hartley, 2008; Wilcken, 2008; Nadel et al., 2009; Martínez et al., 2009a, b; Ramanagouda et al., 2010; Noack et al., 2011; Garcia et al., 2013), causando daño en el follaje, incluso la defoliación completa del árbol (Noack \& Coviella 2006; Nadel et al., 2009; Martínez et al., 2009a).
Este dañino insecto es originario de los bosques de eucaliptos en la Región Australiana, y por acción humana se ha extendido recientemente a numerosas repoblaciones de estos árboles en otros continentes y lugares tan distantes como Sudáfrica (2003), Zimbawe (2004), Argentina (2005), Chile (2006), Uruguay (2008) y Brasil (2008) (Ide et al., 2001; Jacobs \& Neser 2005; Carpintero \& Dellapé, 2006; Martinez-Crosa, 2008; Wilcken, 2008; Nadel et al., 2009; Martínez \& Bianchi 2010; Wilcken et al., 2010; Savaris et al., 2011; Giliomee, 2011). Hasta ahora, era Italia (Laudonia \& Sasso, 2012) el país europeo donde había sido citado este insecto, y ahora ha sido hallado en Portugal (Garcia et al., 2013), siendo una nueva potencial especie invasora y una posible plaga en las plantaciones de eucaliptos en la Península Ibérica.

No es demasiado extraño el hecho de que, por acción humana, algunas especies pertenecientes a esta familia acaben invadiendo otras zonas geográficas diferentes a las que originariamente pertenecen (ver por ejemplo la distribución de Hemerobius stigma, Micromus variegatus o Wesmaelius subnebulosus ahora citadas), y no parece que este flujo intercontinental de especies de esta familia sea un hecho infrecuente. Ya Klimaszewski \& Kevan (1988b); Monserrat (1996); Klimaszewski et al. (2009), etc., citan especies transportadas de Sudamérica a Norteamérica, o desde la Región Paleártica a la Región Neártica.

Indiscutiblemente la llegada de este neuróptero a Portugal es independiente de la dispersión del citado hemíptero sobre el que aparentemente los ejemplares ahora citados depredaban, o del traslado y repoblación de estos australianos árboles desde su origen nativo, ya que ni uno ni otro proceden del área donde $H$. bolivari es conocida. Esta especie ha sido citada como extremadamente eurioica (Monserrat, 1991), y por ello no está específicamente comprometida con un determinado sustrato vegetal o tipo de presa, y menos a colonias de esta plaga. Sin duda esta especie de neuróptero ha llegado accidentalmente a Portugal con la importación (más probablemente desde Brasil) de elementos vegetales llegados a Lisboa o Setúbal, y que han podido extenderse al citado Instituto de Agronomía, sin que se descarte que haya llegado desde su zona original de distribución (Sudamérica) acompañando a la citada plaga desde poblaciones neotropicales de este insecto invasor.

A pesar de la planta sustrato sobre la que estos ejemplares fueron hallados, está fuera de duda que los ejemplares de Hemerobius bolivari, ahora citados de Portugal, pertenecieran a alguna especie invasora australiana de este género, en particular a Hemerobius australis Walker, 1853, especie conocida de Queensland y New South Wales, con la que difieren en su morfología alar y genitalia masculina (New, 1981, 1988a; Smithers, 1993). 
Por el contrario, sí muestran las características de pigmentación, morfología alar y genitalia masculina que sobre Hemerobius bolivari anotó Monserrat (1996).

Hemerobius bolivari Banks, 1910 representa el segundo caso de especies invasoras en la Península Ibérica pertenecientes a esta familia, en ambos casos en Portugal. La especie Sympherobius gayi Navás, 1910 (= Sympherobius maculipennis Kimmins, 1929) es conocida de Chile, Argentina, Uruguay, Paraguay, Brasil, Bolivia y Perú, y ha sido introducida como agente de control en Nigeria, y accidentalmente o por acción humana en la Isla de Pascua y en Portugal: Monserrat (1991) la cita del Algarve: Patacão, Faro, y de los alrededores de Lisboa: Oeiras; y Monserrat \& Deretsky (1999) de Estremadura: Mafra. En estas poblaciones lusas, esta especie parece ser abundante en plantaciones de Citrus sinensis (Rutaceae) y sobre colonias de Planococcus volvae (Homoptera: Pseudococcidae) sobre Chamaecyparis sp. (Cupressaceae), y por ello estas poblaciones parecen estar aparentemente establecidas (Monserrat, 1991; Monserrat \& Deretsky 1999), y por su euroicidad, es muy probable que se encuentren en fase de expansión. La distribución conocida hasta ahora de estas dos especies en la Península Ibérica se anota en la figura 1.

Material estudiado: PORTUGAL: Lisboa, Lisboa, Tapada da Ajuda, Arboreto del Instituto Superior de Agronomia, 29SMC88 (50 m), V.2012, $10^{7}$ ex 1. capturada sobre hojas de Eucalyptus camaldulensis, VI.2012, $10^{7}$ ex 1. capturada sobre hojas de Eucalyptus sp., ambas larvas sobre colonias de Thaumastocoris peregrinus.

\section{Hemerobius handschini Tjeder, 1957}

Elemento mediterráneo septentrional, probablemente holomediterráneo. Comúnmente asociado a coníferas del género Pinus.

Material ESTUdiado: ESPAÑA: Granada, Ferreira, 30SVG91 (1356 m), 8.V.2011, $10^{7}$ sobre Pinus halepensis, Hoya de Pedraza, 30SVG60 (2000 m), 30.VIII.2009, 1 o sobre P. sylvestris. Huesca, Bielsa, Circo de Pineta, 31TBH62 (1300 $\mathrm{m}), 18$.VII.2009, 1 우 sobre $P$. sylvestris, Neril, Los Paules, Coll de Espina, 31TCH00 (1409 m), 17.VII.2009, $10^{7}$ sobre $P$. sylvestris. Parzán, Hospital, 31TBH73 (1600 m), 18.VII.2009, $10^{7}, 1$ 울 sobre $P$. uncinata. Lérida, Baqueira, Puerto de la Bonaigua, 31TCH92 (1200 m), 16.VII.2009, 1 ㅇ sobre P. uncinata, Sant Joan de Lern, 31TCG69 (1096 m), 15.VII.2009, $30^{7} \sigma^{\pi}$ sobre $P$. sylvestris.

\section{Wesmaelius subnebulosus (Stephens, 1836)}

Especie holártica (probablemente es introducida en Norte América, y sin duda en Nueva Zelanda). Significativamente eurioica.
Material estudiado: ESPAÑA: Barcelona, Barcelona, 31TDF38 (10 m), 10.II.2003, 1 ㅇ dentro de casa, M. Carles-Tolrá. Cádiz, Algodonales, 30STF88 $(350 \mathrm{~m})$, 26.V.2012, $10^{7}$ sobre Quercus rotundifolia, 2 우 $ᄋ$ sobre Pistacea sp., Ubrique, 30STF85 (530 m), 25.V.2012, 1 ᄋ sobre Q. suber. Ciudad Real, Navas de Estena, P.N. de Cabañeros, 30SUJ67 (660 m), 16.V.2012, $20^{7} \sigma^{7}, 2$ 우 $ᄋ$ sobre $Q$. ilex, 17.V.2012, 1 웅 sobre $Q$. faginea. Guipúzcoa, Ataun, Aia, 30TWN65 $(614 \mathrm{~m})$,

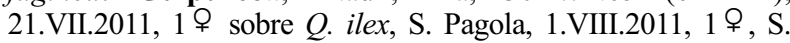
Pagola, Enirio, P. N.Aralar, 30TWN75 (750 m), 11.VI.2007, 1 웅 sobre Fraxinus excelsior, S. Pagola, Oiartzun, Aiko Harria P.N., 30TWN98 (600 m), 11.VI.2007, 1 ㅇ, S. Pagola. Huelva, La Corte, 29SPB99 $(520 \mathrm{~m}), 23 . \mathrm{V} .2012,1$ 우 sobre $Q$. rotundifolia. Jaén, Aldeaquemada, 30SVH64 (950 m), 24.VI.2009, $10^{\text {T}}$ sobre Juniperus oxycedrus. Madrid, Abantos, Fuente del Cervunal, 30TVK09 (1684 m), 2.II.2008, $10^{7}$ sobre Pinus sylvestris, Ciudad Universitaria, 30TVK37 (640 m), 9.V.2012, $10^{7}$ sobre Arbutus unedo, El Escorial, Fuente de la Teja, 30TVK09 (1140 m), 21.VI.2008, 1 웅 sobre hierba, 2.VII.2008, 1 운 sobre Acer sp., El Escorial, Arboreto Luis Ceballos, 30TVK09 (1317 m), 217.VII.2008, $10^{\text {th }}, 1$ 을 en Trampa de Malaise, 31.VII.2008, 1 웅 sobre P. pinaster, Getafe, 30TVK36, 21.V.2012, $10^{\text {T }}$ en trampa de Ricino, M.A.Vázquez, Madrid, Campo del Moro, 30TVK37 (600 m), 31.V.2010, 1 क sobre Cedrus, L. Hiernaux. Salamanca, La Alberca, 29TQE48 (1000 m), 27.VI.2008, 1 a la luz. PORTUGAL: Trás os Montes e Alto Duoro, Chaves, Tronco, 29TPG42 $(825 \mathrm{~m}), 25 . \mathrm{VI} .2008,1$ 웅 sobre Q. rotundifolia, Mazouco, 29SPB31 (540 m), 24.VI.2008, $10^{\pi}$ sobre $Q$. robur.

\section{Wesmaelius navasi (Andreu, 1911)}

Especie eremial conocida del límite meridional de la Región Paleártica occidental, desde Madeira y Canarias, sur de Europa, Marruecos y Argelia a Afganistán, Pakistán y Mongolia. De biología muy poco conocida, asociada a medios térmicos y xéricos.

Material estudiado: ESPAÑA: Almería, Almería, 30SWF57 (10 m), 2.I.1992, 1 ㅇ, J. L. Gómez, Punta Entina Sabinar, 15.IX.2013, $10^{7}, 1$ 우 a la luz, Rambla del Retamar, 13.IX.2013, $1 \mathrm{~m}$ a la luz.

\section{Wesmaelius ravus (Withycombe, 1923)}

Especie holártica de distribución fragmentaria postglacial, citada en zonas elevadas (hasta $2400 \mathrm{~m}$ ), desde Europa y Anatolia al Cáucaso y Japón. Aparentemente asociada a coníferas de alta montaña, especialmente a Pinus.

Esta escasa, enigmática y orófila especie fue citada en la Península Ibérica (Sierra de Guadarrama, Madrid, a 1500 y $2100 \mathrm{~m}$ ) por Monserrat $(1977,1978$ b), no existiendo citas posteriores, ya que francamente seguimos dudando enormemente de las "citas" que se sugieren del mapa de distribución que de esta especie en Europa anotan Aspöck et al. (1980): Cataluña, Palma de Mallorca y Galicia, alguna de las cuales nos parece demasiado lito- 
ral y alguna ya fue cuestionada por Monserrat (2005). La presencia de una nueva población en zonas altas de Sierra Nevada (Granada, $2050 \mathrm{~m}$ ) amplía significativamente su distribución en la península, y abre la posibilidad sobre su existencia en otras elevadas formaciones montañosas de esta península.

Material estudiado: ESPAÑA: Granada, Collado de la Sabina, 30SVG60 (2050 m), 30.VI.2011, $10^{\top}$ sobre Pinus sylvestris.

Sympherobiinae Comstock

Sympherobius elegans (Stephens, 1836)

Especie ampliamente distribuida en Europa y extendida hasta Anatolia y Kazajstán. Aparentemente eurioica.

Material estudiado: ESPAÑA: Almería, Adra, 17.VII.2013, 1 우 a la luz, Cáñar, 30SVF68 (1354 m), 18.VII.2011, 1 우 sobre Pinus sylvestris, Collado de la Sabina, 30SVG60 (2050 m), 10.VII.2012, $10^{7}, 2$ 우 우 sobre P. sylvestris. Ciudad Real, Navas de Estena, P.N. de Cabañeros, 30SUJ67 (660 m), 17.V.2012, 1 ᄋ sobre Quercus pyrenaica. Huelva, Castaño de Robledo, 29SQB09 (680 m), 23.V.2012, 1 ㅇ sobre Q. canariensis. León, León, Azucarera, 30TTN81 (838 m), 19.IX.2007, 10 $0^{7}$, M. Delgado. Toledo, San Pablo de los Montes, 30SUJ87 (940 m), 16.V.2012, 1 ㅇ sobre Q. ilex.

\section{Sympherobius pygmaeus (Rambur, 1842)}

Especie de distribución holomediterránea expansiva, desde Canarias hasta Azerbaiján y Kazajstán. Marcadamente eurioica.

MATERIAl estudiado: ESPAÑA: Álava, Izuraitz-Gauna, Gazeo, 30TWN44 (600 m), 25.V.2007, 1 우 sobre Crataegus monogyna, S. Pagola, 15.VII.2007, 1 우 sobre C. monogyna, S. Pagola. Almería, Tabernas, 30SWG50 (259 m), 29.VI.2011, 1 ㅇ sobre Tamarix gallica, 18.VII.2011, 1 우 sobre Tamarix gallica. Badajoz, Río Sillo, 29SQC01 (405 m), 19.VI.2012, 1 Q sobre Quercus rotundifolia. Cádiz, Hozgarganta, Los Alcornocales, 30STF74 (230 m), 21.VI.2012, $10^{7}$ sobre Q. suber, El Bosque, 30STF77 (270 m), 21.VI.2012, 1 Q a la luz, Pinsapar de Grazalema, 30STF87 (770 m), 26.V.2012, 1 ㅇ sobre Abies pinsapo. Ciudad Real, Navas de Estena, P.N. de Cabañeros, 30SUJ67 (660 m), 16.V.2012, $20^{7} \sigma^{7}, 2$ 우 우 sobre $Q$. ilex, 2 우 우 a la luz, 17.V.2012, $4 \sigma^{\top} \sigma^{7}, 1$ 우 sobre $Q$. pyrenaica. Granada, Collado de la Sabina, 30SVG62 (2050 m), 10.VII.2012, $20^{7} \sigma^{7}$, 5 우 우 sobre Pinus sylvestris. Madrid, El Escorial, Abantos, Fuente del Cervunal, 30TVK09 (1684 m), 17.VI.2008, $10^{7}$, San Fernando de Henares, 30TVK57 (600 m), 27.IX.2010, $10^{\text {T, }, ~ J . ~ I . ~}$ López-Colón. PORTUGAL: Alentejo, Elvás, 30TNF75 (324 m), 7.VII.2008, 10", 1 ᄋ sobre Q. rotundifolia.

\section{Sympherobius pellucidus (Walker, 1853)}

Especie mediterránea septentrional. Generalmente citada sobre coníferas.
Material estudiado: ESPAÑA: Huesca, Plan, Coll de Sahún, 31TBH81 (1960 m), 17.VII.2009, 1 ㅇ sobre Abies alba. Lérida, La Bonaigua, Bosque del Gerdar, 31TCH32 (1472 m), 16.VII.2009, 2 우 운 sobre A. alba.

\section{Sympherobius fuscescens (Wallengren, 1863)}

Especie paleártica. Asociada a coníferas.

Material estudiado: ESPAÑA: Ávila, Casillas, 30TUK66 $(1130 \mathrm{~m}), 15 . \mathrm{V} .2011,10^{7}$ sobre Pinus sylvestris, atraído por feromonas de Matsococcus feytaudi, C. Tavares. Granada, Collado de la Sabina, 30SVG60 (2050 m), 30.VI.2011, $10^{7}$ sobre $P$. sylvestris, 25.VII.2013, $1 \sigma^{\pi}$ sobre P. sylvestris. Lérida, Baqueira, Puerto de la Bonaigua, 31TCH32 (1943 m), 16.VII.2009, $2 \sigma^{\prime \prime} \sigma^{7}, 2$ 우 우 y una larva sobre $P$. uncinata, Rubió, Puerto de Cantó, 31TCG59 (1750 m), 15.VII.2009, 1 ㅇ sobre P. sylvestris, Solsona, 31TCG75 (740 m), 12.V.2011, $10^{7}$ sobre $P$. sylvestris, atraído por feromonas de Matsococcus feytaudi, C. Tavares.

\section{Sympherobius fallax Navás, 1908}

Especie de distribución circum-sahariana expansiva, siendo conocida de las zonas xéricas meridionales del Paleártico Occidental, desde la Macaronesia a Pakistán, y desde el sur de la Región Afrotropical a Arabia Saudí y Yemen. De morfología bastante variable, su biología es poco conocida, está asociada a medios xéricos, y ha sido mayoritariamente capturada a la luz, también ocasionalmente sobre Ceratonia, Ficus, Persea, Salix, Solanum, Psidium, Albizzia, Citrus, Hibiscus o gramíneas.

Material eSTUdiado: ESPAÑA: Almería, Retamar, Rambla del Retamar, 30SWF57 (20 m), 12.VII.2012, 5 우 우 a la luz. Barcelona, Barcelona (10 m), 31TDF38, 30.VI.1997, $10^{\top}, 1$ 우 a la luz, M. Carles-Tolrá, 4.VII.1997, 2 우 ㅇ a la luz, M. CarlesTolrá. Granada, Cerro Gordo, 30SWF36 (200 m), 25.VIII.2009, $10^{7}, 3$ 우 우 a la luz, La Herradura, Punta de la Mona, 30SWF36 (100 m), 26.VII.2009, $10^{\pi}, 1$ ㅇ , 16.VIII.2010, $10^{\top}$, 5.IX.2011, $50^{\pi} \sigma^{\pi}, 2$ 우 우, 13.X.2011, 1 우, 12.XI.2011, 1 우, 29.VII.2013, 1 ㅇ , 23.VIII.2013, $10^{\pi}, 19$. IX.2013, 1 우, 1.XI.2013, 1 운 todos a la luz.

\section{Sympherobius riudori Navás, 1915}

Elemento ibérico. Muy escasamente citado y de biología casi desconocida, ha sido capturado a la luz, sobre Juniperus oxicedrus, Pinus halepensis, P. nigra, Quercus ilex, y en madera y suelo de Fagus sylvatica en descomposición. El dato ahora anotado parece seguir aportando datos que lo relacionan con coníferas.

Material estudiado: ESPAÑA: Granada, Aldeire, 30SVG91 (1661 m), 8.V.2011, $10^{7}$ sobre Pinus halepensis. 
Sympherobius klapaleki Zelený, 1963

Especie mediterránea septentrional. Muy poco conocida y escasamente citada.

Material estudiado: ESPAÑA: Guipúzcoa, Ataun, Aia, 30TWN65 (614 m), 24.VI.2011, 1 우 sobre Quercus ilex, S. Pagola.

\section{Microminae Krüger}

\section{Micromus variegatus (Fabricius, 1793)}

Especie paleártica que probablemente ha sido introducida en la British Columbia Canadiense. Habitualmente asociada a medios boscosos y húmedos.

MAterial estudiado: ESPAÑA: Álava, Izuraitz-Gauna, Gazeo, 30TWN44 (600 m), 15.VII.2007, $10^{7}$ sobre Crataegus monogyna, S. Pagola. Barcelona, Cabrils 31TDF49 (135 m), 11.VIII.1998, 1 ㅇ a la luz, M. Carles-Tolrá.

\section{Micromus angulatus (Stephens, 1836)}

Elemento holártico. Su biología es poco conocida, aparentemente eurioica.

Material estudiado: ESPAÑA: Almería, Gérgal, 30SWG40 $(860 \mathrm{~m}), 29$. VI.2011, $10^{7}$ a la luz. Barcelona, Cabrils, 31 TDF49 (135 m), 19.V.1997, 1 @ a la luz, M. Carles-Tolrá, 16.IX.2000, $10^{7}$ a la luz, M. Carles-Tolrá, 24.VIII.2002, 1 우 a la luz, M. Carles-Tolrá. Granada, La Herradura, Punta de la Mona, 30SWF36 (100 m), 2.I.2012, 1 ㅇ a la luz. Madrid, Buitrago 30TVL43 (970 m), 11.VII.2011, $10^{\pi}$ a la luz. Málaga, Algarrobo, Finca La Mayora, 30SVF07 (92 m), 8.V.2007, $10^{\circ}, 6$.VI.2007, 1 우, 18.VII.2007, 1 ㅇ , 12.XII.2007, $10^{7}$, todos sobre vegetación arvense en cultivo de aguacates, José Miguel Vela (IFAPA), Vélez Málaga, Finca La Alegría, 30SVF07 (140 m), 4.VII.2008, $10^{7}$ en trampas de intersección y de caída colgadas en aguacates, José Miguel Vela.

Los datos que ahora anotamos parecen corroborar su vinculación con la vegetación arvense y/o herbácea. La actividad de los imagos de esta especie en la Península Ibérica parece ser bastante amplia, abarcando desde el mes de febrero, Alcalá de Henares (Madrid) (Monserrat, 1993) a noviembre, Los Molinos, Ribas Vaciamadrid (Madrid) (Monserrat, 1986, 2008a). Con estos nuevos datos ahora anotados se desprende actividad de los imagos a lo largo de todo el año, al menos en el sur de España. Sobre este particular, esta especie se une a las otras dos especies ibéricas de esta familia que presentan actividad (como imagos) en los meses de invierno: Wesmaelius navasi (Andreu, 1911) y Sympherobius fallax Navás, 1908. En la bibliografia $W$. navasi ha sido citada a lo largo de todo el año (salvo agosto) en diferentes zonas ibéricas, y en los meses más fríos ha sido recolectada en enero y diciembre en Mojácar (Almería) (Monserrat, 1983), y $S$. fallax ha sido citada en enero de Espinardo (Murcia) (Monserrat, 1986) y en octubre de La Herradura (Granada) (Monserrat, 2004). De ellas anotamos ahora material que apoya, e incluso amplía, esta información anteriormente conocida sobre sus amplias fenologías.

Micromus lanosus (Zelený, 1962)

Elemento europeo extra-mediterráneo. Asociado a medios boscosos y húmedos.

Esta especie es muy poco conocida de la Fauna Ibérica. Únicamente ha sido citada de Huesca y Lérida (Monserrat, 1984 a, 1991, 1993, 1994; Monserrat, Marín \& Díaz Aranda, 1994) y recientemente de Asturias (Ocharán et al., 2012).

Material estudiado: ESPAÑA: Huesca, Bielsa, Circo de Pineta, 31 TBH62 (1300 m), 18.VII.2009, $10^{\pi}$ sobre Corylus avellana, Villanova, Coll de Sahún, 31TBH91 (1300 m), 17.VII.2009, $30^{\pi} \sigma^{\pi}$ sobre C. avellana.

\section{Megalominae Krüger}

Megalomus hirtus (Linnaeus, 1761)

Especie europea extendida hasta Armenia. De biología poco conocida.

Material estudiado: ESPAÑA: Huesca, Javierre, 31 TBH72 (1130 m), 18.VII.2009, $10^{\prime \prime}$ a la luz.

\section{Megalomus pyraloides Rambur, 1842}

Especie atlanto-mediterránea conocida de España, Francia, Italia y Croacia. De biología poco conocida.

Material estudiado: ESPAÑA: Cádiz, El Bosque, 30STF77 (270 m), 25.V.2012, $10^{7}$ a la luz.

\section{Megalomus tineoides Rambur, 1842}

Especie mediterránea expansiva. De biología poco conocida.

Material estudiado: ESPAÑA: Almería, Tabernas, Rambla Roja, 30SWF59 (370 m), 18.VIII.2011, $10^{\pi}$ a la luz, Gérgal, 30SWG40 (860 m), 29.VI.2011, $30^{\circ} \sigma^{7}, 4$ 우 a la luz. Granada, La Herradura, Punta de la Mona, 30SWF36 (100 m), 28.VII.2009, $10^{\pi}$ a la luz. Huesca, Mesón de Puértolas, 31TBH61 (600 m), 19.VII.2009, $10^{7}$ sobre Pinus pinaster. Jaén, Cazorla, Nava del Pino, 30SWG19 (1500 m), 31.VIII.2010, $2 \sigma^{\pi} \sigma^{x}$ a la luz, M. Baena. 


\section{Agradecimientos}

Esta contribución forma parte del Proyecto coordinado de I+D+i Fauna Ibérica (Neuroptera) CGL2010-22267-C07-05 y del PTDC/AGR-CFL/111877/2009 de la FCT. Deseamos agradecer a los numerosos amigos y colegas, y especialmente a J. I. López Colón, S. Pagola, M. Carles-Tolrá, C. Tavares, M. Baena y J. M. Vela, el envío de ejemplares de sus colecciones particulares o de sus centros de investigación.

\section{Referencias}

Aspöck, H., Aspöck, U. \& Hölzel, H., 1980. Die Neuropteren Europas. 2 vols. Goecke \& Evers. Krefeld. t. I 495 pp., t. II 355 pp.

Aspöck, H., Hölzel, H. \& Aspöck, U., 2001. Kommentierter Katalog der Neuropterida (Iinsecta: Raphidioptera, Megaloptera, Neuroptera) der Westpaläarktis. Denisia, 2: 5-606.

Aspöck, U. \& Aspöck, H., 1982. Eine neue Species des Genus Wesmaelius Krüger aus Spanien (Neuropteroidea: Planipennia: Hemerobiidae). Entomologische Zeitschrift, 92(20): 289-292.

Báez, M. \& Oromí, P., 2010. Planipennia. In: M. Arechavaleta Hernández et al. (Coords.). Lista de especies silvestres de Canarias (hongos, plantas y animales terrestres). Gobierno de Canarias. La Laguna: 253-254.

Banks, N., 1910. New South American Neuropteroid Insects. Proceedings of the Entomological Society of Washington, 12: 146-160.

Carpintero, D. L. \& Dellapé, P. M., 2006. A new species of Thaumastocoris Kirkaldy from Argentina (Heteroptera: Thaumastocoridae: Thaumastocorinae). Zootaxa, 1228: 61-68.

Cassis, G., Schuh, R. T. \& Brailovsky, H., 1999. A review of Onymocoris (Heteroptera: Thaumastocoridae), with a new species, and notes on hosts and distributions of other thaumastocorid species. Acta Societatis Zoologicae Bohemoslovacae, 63: 19-36.

Garcia, A., Figueiredo, E., Valente, C., Monserrat, V. J. \& Branco, M., 2013. First record of Thaumastocoris peregrinus in Western Europe and of the neotropical predator Hemerobius bolivari in Europe. Bulletin of Insectology, 66(2): 251-256.

Giliomee, J. H., 2011. Recent establishment of many alien insects in South Africa, a cause for concern. African Entomology, 19(1): 151-155.

Hartley, M., 2008. Thaumastocoris, The bronzing bug. Fact Sheet, The Arborist Network. Disponible en: http://www.arboristnetwork.com.au/Fact_Sheets.html

Hölzel, H., 2007. Hemerobiidae of the Afrotropical region: A review of current knowledge. Annali del Museo civico di Storia Naturale Ferrara, 5: 1-10.

Ide, M. S., Ruiz, G. S., Sandoval, C. A. \& Valenzuela, J., 2001. Detección de Thaumastocoris peregrinus
(Hemiptera: Thaumastocoridae) asociado a Eucalyptus spp. en Chile. Bosque, 32(3): 309-313.

Jacobs, D. H. \& Nser, S., 2005. Thaumastocoris australicus Kirkaldy (Heteroptera: Thaumastocoridae): a new insect arrival in South Africa, damaging to Eucalyptus trees. South African Journal of Science, 101: 233-236.

Kevan, D. K. McE. \& Klimaszewski, J., 1986. Notes on the Hemerobiidae or brown lacewings flies (Neuroptera) of Canada and Alaska. Neuroptera International, 4: 7-22.

Kevan, D. K. McE. \& Klimaszewski, J., 1987. The Hemerobiidae of Canada and Alaska. Genus Hemerobius L. Giornale Italiano di Entomologia, 16: 305-369.

Klimaszewski, J. \& Kevan, D. K. McE., 1985. The brown lacewing flies of Canada and Alaska (Neuroptera: Hemerobiidae). Part I. The Genus Hemerobius Linnaeus: systematics, bionomics and distribution. Memoire (Lyman Entomological Museum and Research Laboratory), 15: iv + 1-119.

Klimaszewski, J. \& Kevan, D. K. McE., 1987a. The brown lacewing flies of Canada and Alaska (Neuroptera; Hemerobiidae). Part II a. The genus Wesmaelius Krüger. Neuroptera International, 4: 153-204.

Klimaszewski, J. \& Kevan, D. K. McE., 1987b. The brown lacewing flies of Canada and Alaska (Neuroptera; Hemerobiidae). Part II b. The genus Wesmaelius Krüger. Neuroptera International, 4: 245-274.

Klimaszewski, J. \& Kevan, D. K. McE., 1988a. New distribution data for Canadian Hemerobiidae (Neuroptera): Genera: Hemerobius Linnaeus, Micromus Rambur and Wesmaelius Kruger. Supplement [1st]. Revue d'Entomology du Québec, 32: 9-15.

Klimaszewski, J. \& Kevan, D. K. McE., 1988b. The brown lacewing flies of Canada and Alaska (Neuroptera: Hemerobiidae). Part III. The genus Micromus Rambur. Giornale Italiano di Entomologia, 19: 31-76.

Klimaszewski, J. \& Kevan, D. K. McE., 1990a. Distribution data for some Nearctic species of the genus Hemerobius Linnaeus and Wesmaelius Krüger (Neuroptera: Hemerobiidae). Supplement 2. Neuroptera International, 5: 205-210.

Klimaszewski, J. \& Kevan, D. K. McE., 1990b. New distribution data for some American species of the genus Hemerobius Linneaus, with a redescription of Hemerobius alpestris Banks (Neuroptera: Hemerobiidae). Annals of the Transvaal Museum, 35:151-155.

Klimaszewski, J. \& Kevan, D. K. McE., 1990c. The genus Micromus Rambur in North America, with special reference to Canada and Alaska, a synopsis (Insecta: 
Neuroptera: Hemerobiidae). In: M. W. Mansell and H. Aspöck, (Eds.). Advances in Neuropterology. Proceedings of the Third International Symposium on Neuropterology. South African Department of Agricultural Development. Pretoria: 63-66.

Klimaszewski , J. \& Kevan, D. K. McE., 1992. Review of Canadian and Alaskan brown lacewing flies (Neuroptera: Hemerobiidae) with a key to the genera. Part IV: The genera Megalomus Rambur, Boriomyia Banks, Psectra Hagen and Sympherobius Banks. Annals of the Transvaal Museum, 35: 435-457.

Klimaszewski , J., Lesage, L. \& Savard, K., 2009. First record of adventive species Micromus variegatus (Fabricius) from eastern Canada (Neuroptera, Hemerobiidae). Zookeys, 27: 1-6.

Lara, R. I. R. \& Freitas, S. de, 2003. Caracterização morfológica de espécies de Hemerobius Linnaeus, 1758 (Neuroptera: Hemerobiidae) associadas a cultivos de café (Coffea arabica L.), milho (Zea mays L.) e ervamate (Ilex paraguariensis St.Hill.). Revista Brasileira de Entomología, 47(3): 427-434.

Laudonia, S. \& Sasso, R., 2012. The bronze bug Thaumastocoris peregrinus: a new insect recorded in Italy, damaging to Eucalyptus trees. Bulletin of Insectology, 65(1): 89-93.

Makarkin, V. N., 1990. A check-list of the NeuropteraPlanipennia of the USSR far East, with some taxonomic remarks. Acta Zoologica Hungarica, 36: 37-45.

Makarkin, V. N., 1993. The brown lacewings from Vietnam (Neuroptera Hemerobiidae). Tropical Zoology, 6: 217-226.

Makarkin, V. N. \& Monserrat, V.J., 2007. Notes on the identity of Hemerobius amurensis Navás, 1929 (Neuroptera, Hemerobiidae). Deutsche Entomologische Zeitung, 54, 2: 267-270.

Marín, F. \& Monserrat, V. J., 1995. Contribución al conocimiento de los neurópteros de Zaragoza (Insecta, Neuropteroidea). Zapateri, 5: 109-126.

Martínez, G. \& Bianchi, M., 2010. Primer registro para Uruguay de la chinche del eucalipto, Thaumastocoris peregrinus Carpintero y Dellappé, 2006 (Heteroptera: Thaumastocoridae). Agrociencia, 14(1): 15-18.

Martínez, G., Núñez, P., González, W., Rodríguez, F. \& Gómez, M., 2009a. Distribución vertical de la chinche del eucalipto Thaumastocoris peregrinus Carpintero y Dellapé 2006 (Hemiptera; Thaumastocoridae): Resultados preliminares. In: INIA (Eds.). Jornada Técnica, Prospección Forestal. Canelones: 31-35.

Martínez, G., Simeto, S. \& Balmelli, G., 2009b. La chinche del Eucalipto en Uruguay: Panorama de la Investigación a un Año de su Detección Oficial. Revista INIA, 18:33-35.

Martínez-Crosa, G., 2008. Thaumastocoris peregrines Carpintero \& Delappé, (Heteroptera: Thaumastocoridae): new pest found in eucalyptus in Uruguay. In:
IUFRO Recent Advances in Forest Entomology, Pretoria, South Africa: 32-33.

McEwen, P., New, T. R. \& Whittington, A. E., 2001. Lacewings in the Crop Environment, Cambridge University Press. Cambridge. 546 pp.

Monserrat, V. J., 1976a. Una nueva especie del género Sympherobius en España (Neuroptera s.str., Hemerobiidae). Nouvelle Revue d.Entomologie, 6(2): 199-203.

Monserrat, V. J., 1976b. Un nuevo Hemeróbido de España (Neuroptera, Planipennia, Hemerobiidae). Bulletin de la Société d'Histoire Naturelle de Toulouse, 112(1-2): 16-22.

Monserrat, V. J., 1977. Los Neurópteros del Guadarrama. Trabajos de la Cátedra de Artrópodos. Facultad de Biologia, Universidad Complutense, 19: 1-202.

Monserrat, V. J., 1978a. Contribución al conocimiento de los Neurópteros de Orense (Neu. Planipennia). Boletín de la asociación Española de Entomología, 2: 169-184.

Monserrat, V. J., 1978b. Kimminsia rava (Withycombe 1923) nueva para la fauna española (Insecta, Planipennia, Hemerobiidae). Miscellanea Zooogica, 4(2): 177-181.

Monserrat, V. J., 1980. Sobre las especies africanas del género Notiobiella Banks, 1909 (Neuroptera, Planipennia, Hemerobiidae) I. Neuroptera International, 1(1): 26-33.

Monserrat, V. J., 1983. Sobre los Neurópteros de las Islas Canarias, IV: Wesmaelius (Kimminsia) navasi (Andreu, 1911). (Neur., Plan. Hemerobiidae). Boletín de la asociación Española de Entomología, 6(2): 209-224.

Monserrat, V. J., 1984a. Contribución al conocimiento de los neurópteros de Huesca (Neuroptera, Planipennia). Pirineos, 121: 29-50.

Monserrat, V. J., 1984b. Sobre las Especies Africanas del Género Notiobiella Banks, 1909, II. (Neuroptera, Planipennia, Hemerobiidae). In: J. Gepp (Ed.). Progress in World's Neuropterology, Proceedings of the $1^{\text {st }}$ International Symposium on Neuropterology, Graz: 99-124.

Monserrat, V. J., 1985. Lista de los tipos de Mecoptera y Neuroptera (Insecta) de la colección L. Navàs, depositados en el Museo de Zoología de Barcelona. Miscellània Zoològica, 9: 233-243.

Monserrat, V. J., 1986. Sinopsis de los hemeróbidos de la Península Ibérica (Neuroptera, Planipennia, Hemerobiidae). Actas de las VIII Jornadas de la Asociación española de Entomología, Sevilla: 12001223.

Monserrat, V. J., 1990a. A Systematic checklist of the Hemerobiidae of the world (Insecta: Neuroptera). In: M. W. Mansell \& H. Aspöck (Eds.): Advances in Neuropterology. Proceedings of the Third Interna- 
tional Symposium on Neuropterology. Pretoria: 215262.

Monserrat, V. J., 1990b. Systematic studies on Hemerobiidae (Insecta: Neuroptera). In: M. W. Mansell \& H. Aspöck (Eds.): Advances in Neuropterology. Proceedings of the Third International Symposium on Neuropterology. Pretoria: 67-88.

Monserrat, V. J., 1990c. Some brown lacewings from Equatorial Guinea (Neuroptera, Planipennia: Hemerobiidae). Journal of African Zoology, 104: 1722.

Monserrat, V. J., 1990d. Revisión de la obra de L. Navas, II: El Género Micromus Rambur, 1842 (Neuropteroidea, Planipennia: Hemerobiidae). Graellsia, 46: 175-190.

Monserrat, V. J., 1991. Nuevos datos sobre los hemeróbidos ibéricos (Insecta, Neuroptera: Hemerobiidae). Zoologica Baetica, 2: 101-113.

Monserrat, V. J., 1992a. New data on the Afrotropical brown lacewings (Neuroptera: Hemerobiidae). Journal of the entomological Society of Southern Africa, 55(1): 123-136.

Monserrat, V. J., 1992b. On some African species of the genus Micromus Rambur, 1842 (Insecta: Neuroptera: Hemerobiidae). In: M. Canard, H. Aspöck \& M. W. Mansell (Eds.). Proceedings of the Fourth International Symposium on Neuropterology, Toulouse: 271-278.

Monserrat, V. J., 1993. New data on some species of the genus Micromus Rambur, 1842 (Insecta: Neuroptera: Hemerobiidae). Annali del Museo Civico di Storia Naturale "G. Doria", 89: 477-516.

Monserrat, V. J., 1994. Nuevos datos sobre las especies de hemeróbidos ibéricos (Neuroptera: Hemerobiidae). Boletín de la Asociación española de Entomología, 18(3-4): 9-25.

Monserrat, V. J., 1996. Revision del genero Hemerobius de Latinoamérica (Neuroptera, Hemerobiidae). Fragmenta entomologica, Roma, 27(2): 399-523.

Monserrat, V. J., 1998. Nuevos datos sobre los hemeróbidos de América (Neuroptera, Hemerobiidae). Journal of Neuropterology, 1: 109-153.

Monserrat, V. J., 2000. Revisión del Género Nusalala (Neuroptera, Hemerobiidae). Fragmenta entomologica, Roma, 32(1): 83-162.

Monserrat, V. J., 2001. New data on the Brown Lacewings from Asia (Neuroptera, Hemerobiidae). Journal of Neuropterology, [2000], 3: 61-97.

Monserrat, V. J., 2002. Family Hemerobiidae. In: N. D. Penny (Ed.). A Guide to the Lacewings (Neuroptera) of Costa Rica. Proceedings of the California Academy of Sciences, 53(12): 238-251, 398-418.

Monserrat, V. J., 2003. Contribución al conocimiento de los hemeróbidos de Patagonia y Tierra del Fuego (Insecta, Neuroptera: Hemerobiidae). Graellsia, 59(1): 37-56.

Monserrat, V. J., 2004. Nuevos datos sobre algunas especies de hemeróbidos (Insecta: Neuroptera: Hemerobii- dae). Heteropterus Revista de Entomología, 4: 1-26.

Monserrat, V. J., 2005. Catálogo de los Neurópteros de Baleares con nuevos datos sobre su fauna (Insecta, Neuroptera). Bolleti de la Societat d'Història Natural de les Balears, 48: 71-85.

Monserrat, V. J., 2008a. Nuevos datos sobre algunas especies de hemeróbidos (Insecta, Neuroptera, Hemerobiidae). Graellsia, 64(2): 233-253.

Monserrat, V. J., 2008b. Sobre la morfología y la ultraestructura del huevo en algunas especies de Hemerobiidae (Insecta, Neuroptera). Graellsia, 64(2): 265-273.

Monserrat, V. J., 2010. Nuevas o interesantes citas de neurópteros en la Península Ibérica (Insecta: Neuroptera). Heteropterus Revista de Entomología, 10(1): 19-34.

Monserrat, V. J., 2011. Sobre algunas especies de neurópteros de la Península Ibérica y de las Islas Canarias de posición taxonómica problemática o con citas dudosas o cuestionables (Insecta, Neuroptera: Megaloptera, Planipennia). Boletín de la Sociedad Entomológica Aragonesa, 49: 123-144.

Monserrat, V. J. \& Deretsky, Z., 1999. New Faunistical, Taxonomic and Systematic Data on Brown Lacewings (Neuroptera:Hemerobiidae). Journal of Neuropterology, 2: 45-66.

Monserrat, V. J. \& Marín, F., 1996. Plant substrate specificity of Iberian Hemerobiidae (Insecta: Neuroptera). Journal of Natural History, 30: 775-787.

Monserrat, V. J., Marín, F. \& Díaz-Aranda, L., 1994. Contribución al conocimiento de los neurópteros de Lérida (Insecta, Neuroptera). Zoologia Baetica, 5: 41-64.

Monserrat, V. J. \& Triviño, V., 2013. Atlas de los neurópteros de la Península Ibérica e Islas Baleares (Insecta, Neuroptera: Megaloptera, Raphidioptera, Planipennia) / Atlas of the Iberian and Balearic lacewings (Insecta, Neuroptera: Megaloptera, Raphidioptera, Planipennia). Monografías de la Sociedad Entomológica Aragonesa, vol. 13, Zaragoza. 154 pp., 136 mapas.

Nadel, R. L., Slippers, B., Scholes, M. C., Lawson, S. A., Noack, A. E., Wilcken, C. F., Bouvet, J. P. \& Wingfield, M. J., 2009. DNA bar-coding reveals source and patterns of Thaumastocoris peregrines invasions in South Africa and South America. Biological Invasions, 12(5): 1067-1077.

New, T. R., 1981. Redescription of Hemerobius australis Walker (Neuroptera, Hemerobiidae). Australian entomological Magazine, 7(6): 97-99.

New, T. R., 1988a. A Revision of the Australian Hemerobiidae (Insecta: Neuroptera). Invertebrate Taxonomy, 2: 339-411.

New, T. R., 1988b. Hemerobiidae (Insecta: Neuroptera) from New Guinea. Invertebrate Taxonomy, 2: 605-632. 
Noack, A. E., Cassis, G. \& Rose, H. A., 2011. Systematic revision of Thaumastocoris Kirkaldy (Hemiptera: Heteroptera: Thaumastocoridae). Zootaxa, 3121: 160.

Noack, A. E. \& Coviella, C. E., 2006. Thaumastocoris australicus Kirkaldy (Hemiptera: Thaumastocoridae): first record of this invasive pest of Eucalyptus in the Americas. General and Applied Entomology, 35: 13-14.

Noack, A. E. \& Rose, H. A., 2007. Life-history of Thaumastocoris peregrines and Thaumastocoris sp. in the laboratory with some observations on behaviour. General and Applied Entomology, 36: 27-33.

Ocharán, R., Ocharán, F. J. \& Anadón, A., 2012. Neurópteros de la Reserva de la Biosfera de Muniellos (Asturias, Norte de España) (Neuroptera s. s., Megaloptera y Raphidioptera). Boletín de la Sociedad entomológica Aragonesa, 50: 421-429.

Ohm, P. \& Báez, M., 2004. Planipennia. In: I. Izquierdo Zamora, J. L. Martín Esquivel, N. Zurita Pérez \& M. Arechavaleta Hernández (Eds.). Lista de especies silvestres de Canarias (Hongas, Plantas y Animales Terrestres). Gobierno de Canarias. La Laguna: 207208.

Oswald, J. D., 1988. A Revision of the Genus Sympherobius Banks (Neuroptera: Hemerobiidae) of America north of Mexico with a synonymical list of the world species. Journal of the New York Entomological Society, 96(4): 390-451.

Oswald, J. D., 1990. Revision of the Neotropical Brown Lacewing Genus Nomerobius (Neuroptera: Hemerobiidae). Annals of the Entomological Society of America, 83(1): 18-29.

Oswald, J. D., 1993a. Revision and cladistic analysis of the world genera of the family Hemerobiidae (Insecta: Neuroptera). Journal of the New York Entomological Society, 101(2): 143-299.

Oswald, J. D., 1993b. A new genus and species of brown lacewing from Venezuela (Neuroptera: Hemerobiidae), with comments on the evolution of the hemerobiid forewing radial vein. Systematic Entomology, 18: 363370 .

Oswald, J. D., 1994. A new phylogenetically basal subfamily of brown lacewings from Chile (Neuroptera: Hemerobiidae). Entomologica scandinavica, 25: 295-302.

Oswald, J. D., 1996. A new brachypterous Nusalala species from Costa Rica, with coments on the evolution of flightlessness in brown lacewings (Neuroptera: Hemerobiidae). Systematic Entomology, 21: 343-352.

Ramanagouda, S., Kumari, K., Vastrad, A. S., Goud, B. \& Kulkarni, H., 2010. Potential alien insects threatening eucalyptus plantations in India. Karnataka Journal of Agricultural Sciences, 23(1): 93-96.

Reguilón, C., 2002. Morfología de los estados inmaduros de Hemerobius bolivari (Neuroptera: Hemerobiidae). Revista Sociedad Entomológica Argentina, 61: 63-68.
Savaris, M., Lampert, S., Valle da Silva Pereira, P. R. \& Salvadori, J. R., 2011. Primeiro registro de Thaumastocoris peregrinus para o estado de Santa Catarina e novas áreas de ocorrência para o Rio Grande do Sul, Brasil. Ciência Rural, Santa Maria, 41(11): 1874-1876

Smithers, C. N., 1993. A note on the Megaloptera, Neuroptera and Mecoptera of Tuglo Wildlife refuge, New South Wales. Australian Entomology, 20(2): 67-71.

Wilcken, C., 2008. Percevejo bronzeado do eucalipto (Thaumastocoris peregrinus) (Hemiptera: Thaumastocoridae): Ameaça ás florestas de Eucalipto brasileiras. Alerta Protef, Instituto de Pesquisas e Estudos Florestais. Botucatu. 11 pp.

Wilcken, C., Soliman, E., Nogueira, L., Rodrigues, L., Ribeiro, T., Ferreira, P. \& Rodrigues, R., 2010. Bronze bug Thaumastocoris peregrines Carpintero and Dellapé (Hemiptera: Thaumastocoridae) on Eucalyptus in Brazil and its distribution. Journal of Plant Protection Research, 50(2): 201-205.

Wise, K. A. J., 1993. Species of Micromus (Neuroptera: Hemerobiidae) in New Zealand. Records of the Auckland Institute and Museum, 30: 93-117.

Wise, K. A. J., 2000. Re-evaluation of three species of Australasian Hemerobiidae (Insecta: Neuroptera:). New Zealand Entomologist, 22: 15-21. 